\title{
Optimized CRT Stimulation Based on Ultra-High-Frequency QRS Analysis
}

\author{
Magdalena Matejkova ${ }^{1}$, Jolana Lipoldova ${ }^{1}$, Pavel Leinveber ${ }^{1}$, Josef Halamek ${ }^{2}$, Pavel Jurak ${ }^{2}$, Filip \\ Plesinger $^{2}$, Andrej Nagy ${ }^{1}$, Miroslav Novak ${ }^{1}$ \\ ${ }^{1}$ The International Clinical Research Center, St. Anne's University Hospital, Brno, Czech Republic \\ ${ }^{2}$ The Czech Academy of Sciences, Institute of Scientific Instruments, Brno, Czech Republic
}

\begin{abstract}
The optimization of cardiac resynchronization therapy (CRT) stimulation by ultra-high-frequency ECG (UHF-ECG) is presented.

34 subjects (24 men, aged 65.0 \pm 10.1 ) undergoing the implantation of a CRT_D with an automatic optimizing algorithm for atrioventricular and intraventricular delay. Interventricular delay (IVD) from echocardiography and electrical dyssynchrony (e-DYS) from UHF-ECG were analysed using three different CRT configurations - OFF (stimulation off), SMART (SMART algorithm) and OPT (optimal assessment subjectively determined by the electrical depolarization map from UHF-ECG).

The mean $\pm S T D$ values over visits for IVD [ms] were $50.32 \pm 29.00,29.09 \pm 29.33$ and $25.17 \pm 24.54$ for OFF, SMART and OPT respectively. For e-DYS

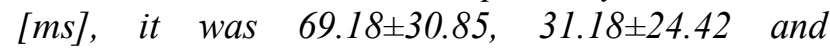
$13.42 \pm 18.67$ for OFF, SMART and OPT respectively. Significant statistical differences $(p<0.01)$ existed between OFF and both SMART and OPT for both IVD and e-DYS parameters. Between SMART and OPT, significant differences $(p<0.01)$ existed only for e-DYS.

UHF-ECG analysis may be used to optimize CRT stimulation and to minimize electrical dyssynchrony.
\end{abstract}

\section{Introduction}

Left ventricular contraction is considered to be synchronous when electrical activation of all ventricular myocardial cells takes less than $120 \mathrm{~ms}$. Ventricular dyssynchrony can be determined at different levels [1]: The early activation of definite left ventricular areas occurs in the presence of intraventricular conduction abnormalities (LBBB). That creates areas of both early and late activation [2]. Intraventricular dyssynchrony means the late activation of the lateral areas of the left ventricle as compared to the interventricular septum. Another level of ventricular dyssynchrony is interventricular dyssynchrony, which means the delayed activation of one chamber compared to the other [1].

In this study, we present the use of ultra-high-frequency ECG (UHF-ECG) [3] to possibly optimize biventricular stimulation CRT (cardiac resynchronization therapy).

\section{Methods}

An ultra-high-frequency high-dynamic-range 14-lead ECG technique (UHF-ECG) and echocardiography were used in the study.

\subsection{Data recording, subjects and study protocol}

34 subjects (24 men and 10 women, aged $65.0 \pm 10.1$ ) undergoing CRT implantation with a device manufactured by Boston Scientific with the possibility to automatically optimize atrioventricular (AVD) and intraventricular (VVD) delay (SMARTdelay algorithm) were included in the study. The patients were evaluated over 48 visits (33 visits were performed the next day after implantation and 15 visits were at the six-month follow up). Each visit consisted of a repeated 3-min recording of a 14-lead ECG (sampling frequency $5 \mathrm{kHz}$ with a dynamic range of 26bits, $2 \mathrm{kHz}$ pass band, M\&I s.r.o., Prague, Czech Republic) for various CRT_D settings while the patient was in a calm supine position with subsequent echocardiography measurements (Vivid E9, GE Healthcare, Wauwatosa, WI) for selected CRT_D settings. The measurements were taken at the International Clinical Research Center at St. Anne's University Hospital, Czech Republic and all subjects signed their informed consent to the investigation.

Interventricular delay (IVD) and electrical dyssynchrony (e-DYS) were used as the numerical parameters. The optimal stimulation was based on electrical depolarization maps that describe the timespatial distribution of electrical activity [3]. The irregularities in electrical activation are best described by these maps. 


\subsection{Processing}

UHF Solver (software developed by ISI AVCR, Brno, Czech Republic) was used to process the data for each UHF-ECG record [4],[5]. The software created an electrical depolarization map for each record. The e-DYS parameter was defined as the difference in the position of normalized maxima (red colour) in leads V1-V8 on the electrical depolarization map.

We subsequently programmed various VVD (left ventricular lead and right ventricular lead stimulation delay) and AVD (atrial lead and right ventricular lead stimulation delay) values of CRT_D settings with the goal of finding subjective optimal settings OPT evaluated based on the appearance of the electrical depolarization map obtained from the UHF-ECG measurements. A thin and straight appearance was considered to be the best result (Figure 1).

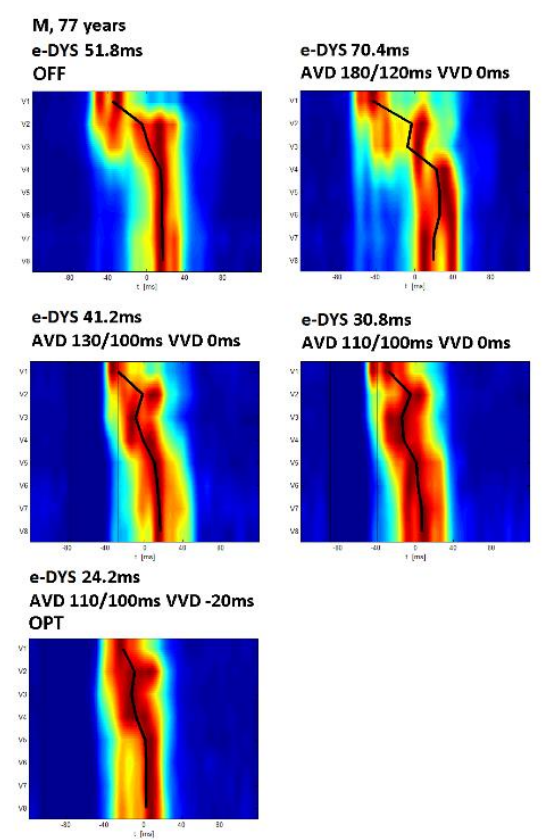

Figure 1. Examples of CRT_D optimization based on the appearance of the electrical depolarization maps.

We tested the settings recommended by the SMART algorithm (SMART) created by the Boston Scientific device. The algorithm was based on measurements taken of the sensing delay between the atrial lead and the right ventricular lead during atrial sensing and pacing and the sensing delay between the right and left ventricular leads. Furthermore, a natural rhythm measurement without stimulation (OFF) was performed (Figure 2).

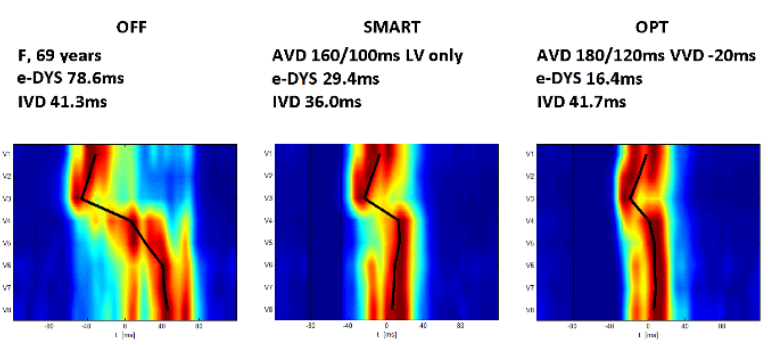

Figure 2. Examples of different CRT_D programming. From the LEFT: OFF (without any stimulation), SMARTdelay settings recommended by device, optimal OPT settings.

Echocardiography was provided for each of these settings (OPT, SMART, OFF). We measured interventricular dyssynchrony (IVD) as the difference between the left and right pre-ejection intervals [2], consisting of the measurement of the aortic pre-ejection time recorded by pulsed-wave (PW) Doppler in an apical 5-chamber view and the pulmonary pre-ejection time recorded in the left parasternal view in the short axis also using PW Doppler [1] (Figure 3).

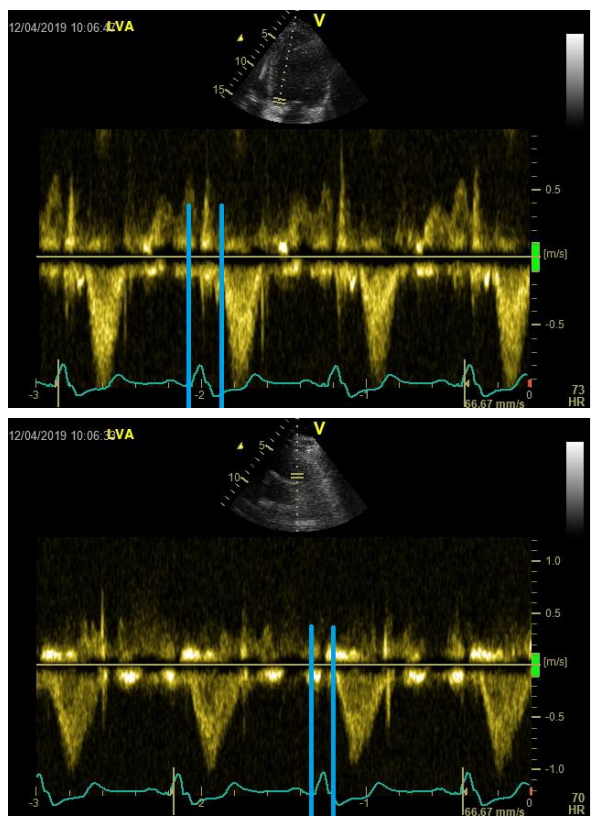

Figure 3. Examples of echocardiography interventricular dyssynchrony measurements. Aortic valve flow at the top, pulmonary valve flow at the bottom (Vivid E9, GE Healthcare).

Each electrical depolarization map was subjectively evaluated using a SUBscore on a scale range of 1 to 5 ( 1 as maximally synchronous activation and 5 as very asynchronous activation) (Figure 4). 


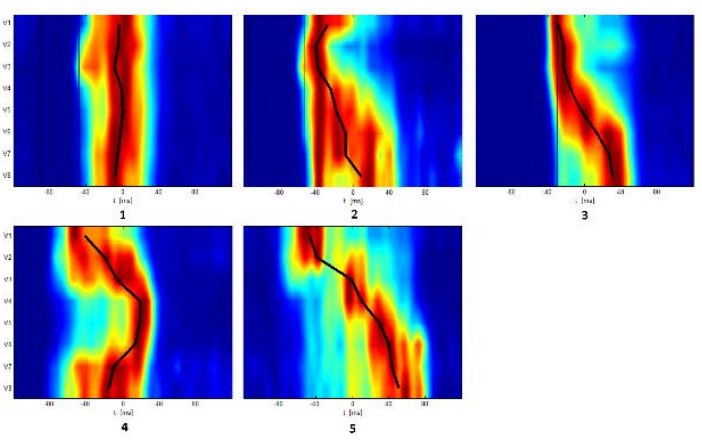

Figure 4. Examples of SUBscores 1 to 5 in different electrical depolarization maps.

\section{Results}

Figure 1 shows examples of possible electrical depolarization maps during our investigation of CRT_D settings. Measurements taken in CRT-OFF mode show typical dyssynchrony.

Table 1. Values (mean \pm std) of interventricular delays IVD and electrical delays e-DYS for different CRT_D settings (OFF, SMART, OPT) and the difference between SMART and OPT values.

\begin{tabular}{lll}
\hline & IVD & e-DYS \\
\hline OFF & $50.32 \pm 29.00$ & $69.18 \pm 30.85$ \\
\hline SMART & $29.09 \pm 29.33$ & $31.18 \pm 24.42$ \\
\hline OPT & $25.17 \pm 24.54$ & $13.42 \pm 18.67$ \\
\hline SMART - OPT & $4.12 \pm 20.61$ & $17.76 \pm 24.96$ \\
\hline
\end{tabular}

The automatic SMARTdelay algorithm determined left ventricular pacing only (with RV pacing off) in $44 \%$ of the cases, biventricular simultaneous pacing (with LV-RV delay $0 \mathrm{~ms}$ ) in $48 \%$ of the cases and biventricular sequential pacing (with nonzero LV-RV delay) in $8 \%$. Atrioventricular delay recommendations were variable.

VVD values of 40/0/-5/-20/-40/-60 ms were used for OPT. Based on the electrical depolarization maps, we set simultaneous biventricular stimulation for $44 \%$ of the cases,

VVD - $20 \mathrm{~ms}$ for $19 \%$ of the cases and VVD $-40 \mathrm{~ms}$ for $21 \%$ of the cases. The most common AVD was $180 / 120 \mathrm{~ms}$ (for postpaced/postsensed configuration) for $52 \%$ of the cases followed by $130 / 100 \mathrm{~ms}$ for $32 \%$ of the cases.
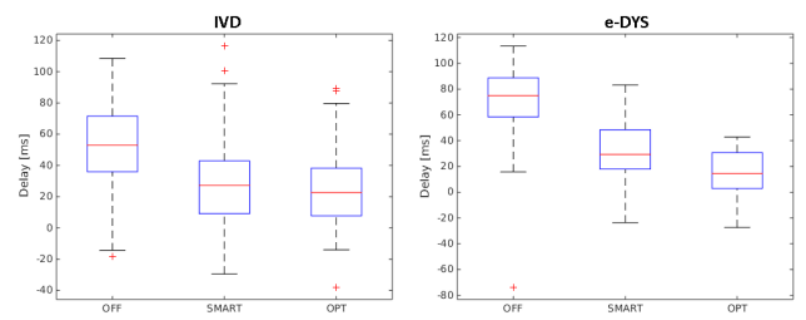

Figure 5. Values of interventricular delays IVD and electrical delays e-DYS for different CRT_D settings (OFF, SMART, OPT)

The IVD parameter decreased by $42 \%$ for SMART and by $50 \%$ for OPT settings in comparison with OFF. For eDYS, there was a 55\% decrease for SMART and an $81 \%$ decrease for OPT (Figure 5).

Significant statistical differences $(p<0.01)$ existed between OFF and both SMART and OPT for both the IVD and e-DYS parameters. Between SMART and OPT, significant differences $(\mathrm{p}<0.01)$ existed only for the e-DYS parameter.

The IVD parameter was smaller for OPT $(-4.12 \pm 20.61)$, but not statistically significant. The difference between the IVD values in groups subdivided by SUBscore and e-DYS were statistically significant (except for 1 versus 2 and 3 versus 4 for SUBscore and except for 1 versus 2 for eDYS). Figure 6 shows the delays of the subdivided groups.
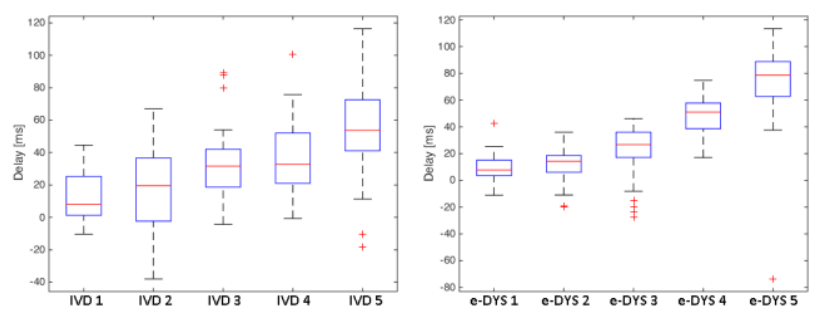

Figure 6. Values of interventricular delays IVD and electrical delays e-DYS subdivided by SUBscore (based on a subjective electrical depolarization map assessment).

\section{Discussion}

The UHF-ECG technique for assessing dyssynchrony is well described in [3]. In this study, we focused on the effect of different CRT_D settings on interventricular (IVD) and e-DYS. The IVD parameter was chosen because it is easier to measure (even with worse investigability), it is highly reproducible and as is shown in [6], the specificity and positive predictive value of the IVD parameter was higher compared to the parameters based on M-mode and tissue Doppler imaging.

Thereafter, we presented the possibility to find the best CRT_D settings depending on electrical depolarization maps (OPT) and recommended SMARTdelay.

As can be seen in [7], there could be some subjects for whom non-profiting from OPT settings and SMART 
settings is more beneficial (12\%). However, the automatic SMARTdelay algorithm recommended non-biventricular stimulation for $44 \%$ of the cases (LV stimulation only), Figure 7.

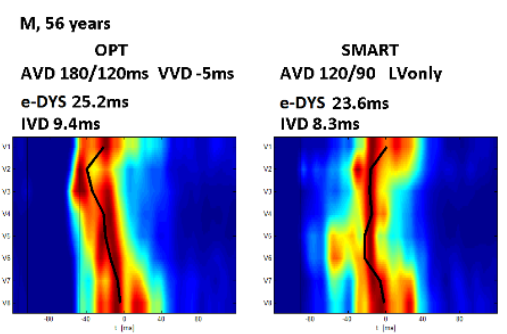

Figure 7. A comparison of numerical parameters and electrical depolarization maps.

We did not set any LV only stimulation (with a straighter appearance of the electrical depolarization map) for optimal settings (OPT) because fusion pacing with spontaneous atrioventricular conduction was not exercise tested [8].

The presence of the stimulation peak directly in the QRS is one limitation of this study. Part of the ultra-highfrequency signal is lost during elimination of the stimulation peak and the results may be distorted.

\section{Conclusion}

UHF-ECG analysis is a suitable method to optimize CRT stimulation and to minimize electrical dyssynchrony. In addition, there is a growing trend to improve mechanical dyssynchrony in comparison with optimization implemented by a device's own algorithm.

\section{Acknowledgments}

Supported was provided by the European Regional Development Fund - Project ENOCH (No. CZ.02.1.01/0.0/0.0/16_019/0000868). Supported by the Czech Science Foundation, project no. GA17-13830S.

\section{References}

[1] S. GHIO et al., "Interventricular and intraventricular dyssynchrony are common in heart failure patients, regardless of QRS duration," Eur. Heart J., vol. 25, no. 7, pp. 571-578, Apr. 2004.

[2] K. Serri, S. Lafitte, R. Amyot, C. Sauve, R. Roudaut, "Echocardiographic evaluation of cardiac dyssynchrony.," Can. J. Cardiol., vol. 23, no. 4, pp. 303-10, Mar. 2007.

[3] P. Jurak et al., "Ventricular dyssynchrony assessment using ultra-high frequency ECG technique," J. Interv.
Card. Electrophysiol., vol. 49, no. 3, pp. 245-254, Sep. 2017.

[4] F. Plesinger, J. Jurco, J. Halamek, P. Jurak, "SignalPlant: an open signal processing software platform," Inst. Phys. Eng. Med. Print. UK Physiol. Meas, vol. 37, pp. 38-48, 2016.

[5] F. Plesinger, J. Jurco, P. Jurak, J. Halamek, "Robust multichannel QRS detection," Computing in Cardiology, vol. 41, pp. 557-560, 2014.

[6] J. Lipoldova, J. Meluzn, M. Novak, E. Ozabalova, "Are our criteria for selection of candidates for cardiac resynchronization therapy correct?" Cor Vasa, vol. 53, pp. 535-541, 2011

[7] P. Jurak, M. Matejkova, J. Halamek, F. Plesinger, I. Viscor, V. Vondra, J. Lipoldova, M. Novak, R. Smisek, P. Leinveber, "Cardiac Resynchronization Guided by Ultra-High-Frequency ECG Maps," Computing in Cardiology, 2019.

[8] R. Vatasescu et al., "Midterm 'super-response' to cardiac resynchronization therapy by biventricular pacing with fusion: insights from electro-anatomical mapping.," Europace, vol. 11, no. 12, pp. 1675-82, Dec. 2009.

Address for correspondence.

Magdalena Matejkova

St. Anne's University Hospital, ICRC

Pekarska 53, Brno, 65691, Czech Republic

magdalena.matejkova@fnusa.cz 